\title{
Calibration and Conformity in the Conductivity Channels of Pharmaceutical Waters Systems: A review
}

\author{
Adolfo Castillo Caballero ${ }^{1}$, German Muñiz Jimenez ${ }^{1}$, Idania Caballero Torres ${ }^{2}$, Luis A Calvo Diaz ${ }^{1}$ and Maria K \\ Martinez Muné ${ }^{3}$
}

${ }^{1}$ Engineering Services Department, Center of Molecular Immunology, Cuba

${ }^{2}$ Knowledge Management Department, Center of Molecular Immunology, Cuba

${ }^{3}$ Virology Department, Institute of Tropical Medicine "Pedro Kourí", Cuba

Submission: June 26, 2018; Published: July 24, 2018

*Corresponding author: Adolfo Castillo Caballero, Engineering Services Department, Center of Molecular Immunology, Cuba, Email: adolfoc@cim.sld.cu

\begin{abstract}
Conductivity is an indicator of the quality in pharmaceutical water. Water systems are divided in two main groups of quality degree PW and WFI. There are two mainly ways to measure conductivity online and offline but the first is the one used for these systems. According with the GMP compliance, water systems are regularly subjected to validation processes, where the calibration of conductivity channel is a mandatory practice for performance qualification. Temperature effects must be considered when calibrating conductivity measuring channels. The conformity assessment in the calibration of the PW and WFI systems is proposed as criteria. As result the guidelines and monographies about all these topics are harmonized. An equipment or system of measurement to be reliable requires a conformity assessment with a probability greater than or equal to $95 \%$ for pharmaceutical industry.
\end{abstract}

Keywords: Calibration, Conductivity, Conformity, Pharmaceutical Water, Uncertainty

Abbreviations: GMP: Good Manufacturing Practice; EP: European Pharmacopoeia; CRM: Certified Reference Material; SS: Standard Solution; ISO: International Organization for Standardization; NIST: National Institute of Standards and Technology; PW: Purified Water; WFI: Water for Injection; RO: Reverse Osmosis; USP: United States Pharmacopeia

\section{Introduction}

The current regulations demand that the production of medicines guarantees high standards of quality, safety and efficacy. In production processes, water with a high degree of purity is considered a critical service [1]. In each technological process, all the measurement operations are critical to characterize the efficiency, quality and safety of the technological processes of all productive operations [2]. Although the different monographs issued by quality regulatory agencies describe several types of water for pharmaceutical use, overall, the employment at an industrial scale of 2 fundamental levels of quality stands out: $\mathrm{Pu}$ rified Water (PW) and Water for Injection (WFI). The water of pharmaceutical quality is incorporated throughout the processes to be part of the final product and is also used in the cleaning of containers and accessories that have direct contact with intermediate products and the product itself, so it must meet chemical and microbiological specifications established in internationally recognized monographs.
The GMP regime requires to maintain a strict metrological control over the equipment and measurement systems of its facilities [3]. Its main objective is to achieve therapeutic products with high levels of safety and efficacy aimed primarily at patients with cancer and other non-communicable chronical diseases. Due to the importance of the quality of pharmaceutical waters, it is urgent to control their variables. One of the indicators to measure water quality is electrolytic conductivity. Significant changes can be indicators of contamination. The most important source of contamination in the pharmaceutical industry is the water used for the manufacture of products $[4,5]$. The instrument responsible for measuring conductivity is the conductometers, so in order to this instrument to make correct measurements must be properly calibrated. The calibration of the equipment has become a key requirement for many quality standards among these GMP and Good Laboratory Practices (GLP) stand out. Obtaining a reliable measurement result is only possible if the elements that constitute the technical bases that 
establish confidence in the measurement are met. One of these elements is the evaluation of uncertainty, an attribute closely related to the concepts of accuracy, traceability, precision, error and tolerance.

Inaccuracy in a measurement may result in the acceptance of a certain number of products that would be out of tolerance, or the rejection of others that would fit within the tolerance range. In practice, a security criterion is chosen that consists in rejecting any measuring in a doubtful situation. Currently, new concepts are introduced, such as measurement capacity and probability of conformity of the measurement, which constitute an effective tool when giving an opinion on the conformity of the calibration performed [2]. The purpose of the conformity assessment is to demonstrate that the conductivity measurement channel, when operating within the established limits (acceptance intervals), produces a product of specified consistency and quality with a high degree of safety and reliability. The main objective of this work is to review the guides and monographs of the regulatory agencies, as well as to standardize the criteria issued by the different pharmacopoeias and that are related to the calibration of the online conductimeters of the pharmaceutical water systems.

\section{Conductivity Measurement}

At a glance, the result of measurement is only an approximation of the real value in a variable, an $100 \%$ accurate measurement is not then possible, and only is complete when is joined to an uncertainty definition [6]. Measuring water conductivity is a well-established way to evaluate its quality [7]. Conductivity is defined as the capacity of any substance to conduct electricity, is the ability of a solution to allow a current of electrical nature to flow from one electrode to another through itself. The basic unit for measuring conductivity is Siemens per centimeters [8]. Conductivity is dependent of many different factors such as: concentration, ions mobility, ions valence and temperature $[8,9]$. Electrical conductivity in water can be seen as a flow of electrons due to the presence of ions. These ions and resulting conductivity can be considered intrinsic to water $[10,11]$. Pure water barely conducts electricity, ions with positive and negative charges are responsible for electrical conduction, so the amount of current conducted relays only in the number of ions and its mobility. If such effect continues until the solution is full of ions, motion capacity is restricted and conductivity may diminish instead of rising, giving cases in which two different concentrations show the same conductivity. Some substances ionize more completely than others and that is why conduct better electrical currents. Every acid, base or salt has its own curve of concentration vs conductivity [12].

Electrical conductivity is an inverse function of resistivity $(\rho)$, measured between two opposite faces in a cube of $1.0 \mathrm{~cm} 3$ volume of a water solution to specific temperature. This solution behaves as an electric conductor, so physics laws of electrical resistance can be applied. In real life conductivity is not measured between two equals electrodes of $1 \mathrm{~cm}$, instead is measured in electrodes with different sizes and shapes, usually rectangular or cylindrical, for this reason when doing the measurement, instead of the conductivity, the conductance is measured, which is then multiplied for a constant from the cell and gives conductivity (Table 1) [13].

Table 1: Cell constants and measurement intervals [13].

\begin{tabular}{|c|c|}
\hline Intervals $(\boldsymbol{\mu S} / \mathbf{c m})$ & Cell Constant $\mathbf{( c m - 1 )}$ \\
\hline 0,05 a 20 & $0,01^{*}$ \\
\hline $1 \mathrm{a} 200$ & $0,1^{*}$ \\
\hline $10 \mathrm{a} 2000$ & 1 \\
\hline $100 \mathrm{a} 20000$ & 10 \\
\hline $1000 \mathrm{a} 200000$ & 50 \\
\hline
\end{tabular}

*used for PW and WFI measurements, due to the fact that they produce low resistance to current flow in high density waters.

There are two ways to measure; on line and off-line conductivity. To measure online in WFI and PW, which typical values varies from $0.055055 \mu \mathrm{S} / \mathrm{cm}$ and $2.0 \mu \mathrm{S} / \mathrm{cm}(18.2-0.5 \mathrm{M} \Omega$ $\mathrm{cm}$ ), the measure is taken in production loop or in the supply tank. Once this water is taken from the distribution system and is stored in a clean recipient and moved to the lab then measure is offline, and conductivity rises from 0.8 to $1.2 \mu \mathrm{S} / \mathrm{cm}$ over its online value (e.g. Figure 1) due to contact with air and its reaction with $\mathrm{CO} 2$, even in clean areas. Samples taken offline have more variability among each other's (noise is not due to measurement is due to purity), so online measurement is the only way to detect little changes [14].

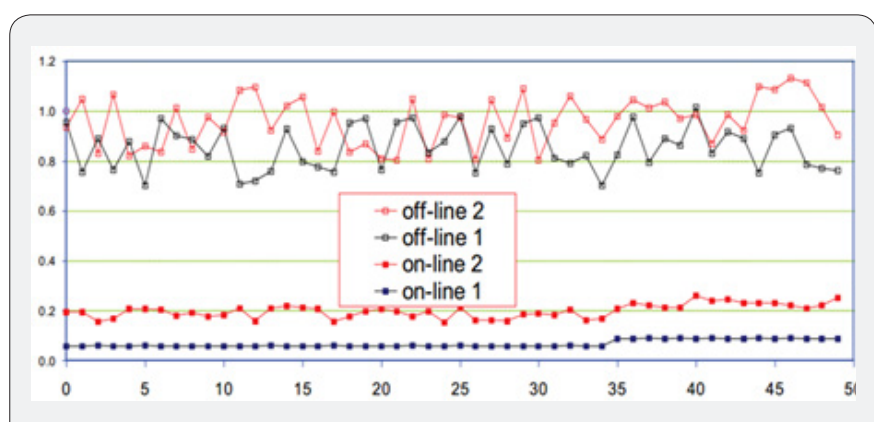

Figure 1: Comparison of on-line and off-line conductivity measurements of two pharmaceutical waters samples [14]. An example based on a METTLER TOLEDO Thornton study.

\section{Temperature and Compensation}

When measuring conductivity is very important to consider temperature, at increase this one conductivity increases too. A rise in temperature is translated in a decrease of viscosity which permits the ions to move faster, increasing its capacity to conduct electricity. The effect of temperature is different for each ion, but as a rule for water solutions the increment is between 1.5 and $5.0 \%$ for every ${ }^{\circ} \mathrm{C}$ [15]. Due to this dependency is needed 
compensation of temperature for measuring conductivity in PW and WFI. Variation and dependency is not linear with conductivity, this is why compensation varies everywhere from $1.5 \%$ / ${ }^{\circ} \mathrm{C}$ to $7 \%$ / ${ }^{\circ} \mathrm{C}$ depending of temperature, concentration and kinds of impurity [15]. Concept of reference temperature (20 or $25 \stackrel{\circ}{\circ}$ ) was created to compare results of conductivity in different temperatures. To make temperature compensation exists different choices or algorithms $[9,16]$
a. Linear function.
b. Non-linear function for natural waters according to ISO/DIN7888.

c. No correction.

d. For Linear compensation the applied equation would be $[9,17]$ :

$$
K_{\text {tref }}=\frac{100}{100+e\left(T-T_{\text {ref }}\right)} \times K_{T}
$$

Where; $\mathrm{k}_{\text {Tref }}$ is conductivity to reference temperature, $\mathrm{k}_{\mathrm{T}}$ is real conductivity, $\mathrm{T}_{\text {ref }}$ is reference temperature, $\mathrm{T}$ is sample temperature (see Figure 2) y $\theta$ is temperature coefficient (acids $1.0-1.6 \% /{ }^{\circ} \mathrm{C}$, bases $1.8-2.2 \% /{ }^{\circ} \mathrm{C}$, salts $2.2-3.0 \% /{ }^{\circ} \mathrm{C}$, etc.) $[9,16]$.

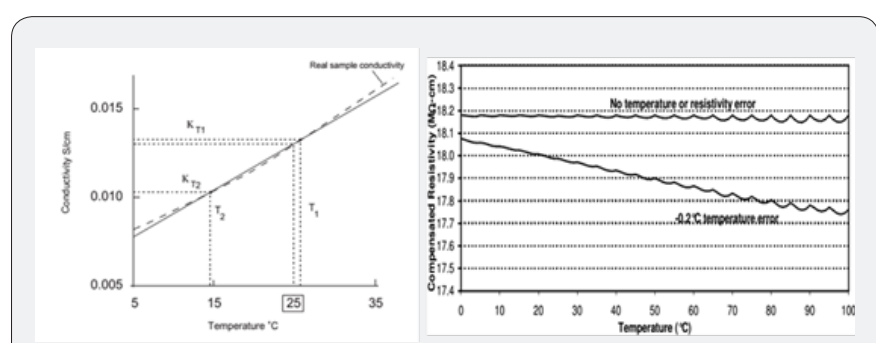

Figure 2: The error increase for high temperatures and the correction is only accurate within the limits T1 and T2. Differences between $T$ and Tref is the biggest risk of error, this could be an uncertainty source [9].

Temperature correction by non-linear ways is not convenient for many water-based liquids, dependency with temperature may be described by non-linear functions [9].

$$
K_{25}=f_{25}(T) \times k T(2)
$$

Where $f_{25}(T)$ is correction factor of temperature for conductivity in water at $25{ }^{\circ} \mathrm{C}$ (conductimeter computes a 4-degree polynomial and adjusts variations of conductivity versus temperature declared in ISO/DIN). When measuring is done under precepts USP 40 and EP 9th edition is not needed temperature compensation. In other hand many current publications read that in order to accomplish effectivity in process supervision when temperature varies, temperature compensation is needed to reveal changes in temperature and purity [15]. To have a "temperature-dependent vs conductivity table" limits and eliminates the temperature compensation effect. This would allow the use of on-line instrumentation to monitor for water systems which temperatures are not at $25^{\circ} \mathrm{C}$.

\section{Conductivity measurement channel}

Any typical conductimeter applies an altern current (I) with an optimal frequency $(94 \mathrm{~Hz}$ in intervals from $4.000 \mu \mathrm{S}$ to $40.00 \mu \mathrm{S}$ and $46.9 \mathrm{kHz}$ from $400.0 \mathrm{mS}$ to $2.000 \mathrm{~S}$ ) in order to activate two electrodes and to measure voltage (V). Both variables, current and voltage, are used to compute conductance (I/V), see Figure 3. So conductimeter measures conductance and the screen reveals conversion from the measured value into conductivity, to carry out a calibration process is needed to know the cell constant value used [18].

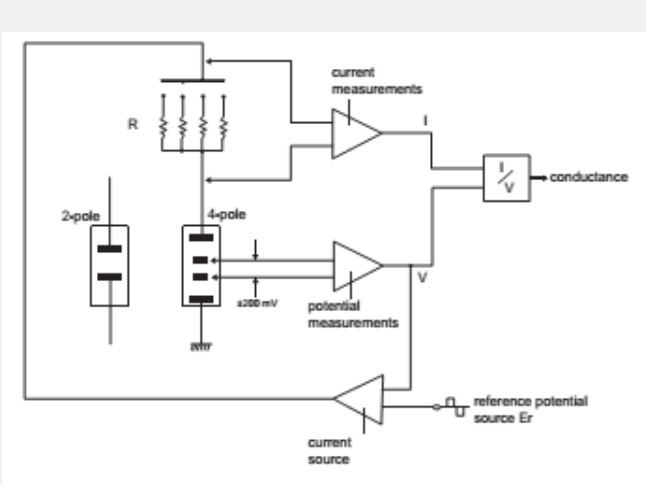

Figure 3: Simple Conductimeter design [9].

There are two main groups of conductimeters: table conductimeters (offline) and online conductimeters, used in different environments and situations. First one is used to measure analytical samples contained on recipients in labs, meanwhile online conductimeters are used in industrial process at big scale productions. Pharmaceutical companies invest a good piece of their capital in the instrumentation needed to guarantee production and distribution of water with good quality and especially in calibrate and certificate these processes [14].

Measuring online conductivity provides real time information and the opportunity to control accurately this variable [11]. Conductimeter measures temperature at the same time, and then converts to reference temperature using correction equations, these readings are referenced at specific temperatures normally $20^{\circ} \mathrm{C}$ or $25{ }^{\circ} \mathrm{C}$ [9]. However, calibration on this instrument must be carried out without temperature compensation and at temperatures near to environment conditions, even so many authors recommend calibrating at higher temperatures to prevent possible errors [13].

A conductivity measurement system is generally composed of two basic elements, conductimeter (analyzer) and electrode. Each of these elements adds uncertainty and must be evaluated each one separated and then together to obtain a combined uncertainty of the calibration process.

\section{Waters for Pharmaceutical and Biotechnology Use:}

\section{Applications}

Water is an excellent solvent and medium for most ways of life on planet. This is the reason why it can pollute so easily at 
contact with almost everything microorganisms grow so well on it, and it is also what makes purification processes so complex that even selecting the system to be used can be a real challenge. Drinking water is the raw material for all typical waters used in pharmaceutical industry, usually purified water or water for injection. Pharmaceutical water systems are considered critical because of their wide use as excipients in pharmaceutical productions. While water quality has a direct potential impact on patients, it is the operation of the purification process that needs to be controlled, and hence there is also a direct relationship between on-line or off-line measurement methods based on analytical methods and its purposes $[15,19]$. Various requirements and monitoring in the purification process such as the conductivity and temperature before distribution are very important for analyzes during and after production $[13,19]$. The purification process has pretreatment phase and purification phase, as can be seen in Figure 4.

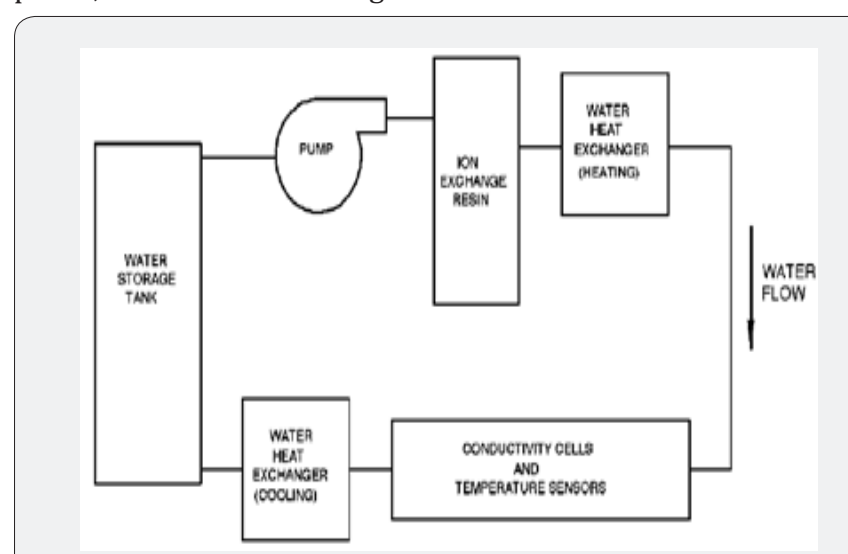

Figure 4: Flow Schematic of Typical Pharmaceutical Water Manufacturing [19].

Each of these steps has its specific design, operation and maintenance characteristics [6]. The quality attributes of both waters differ only in the existence of a requirement related to bacterial endotoxins. The critical difference consists in the control degree of the system and in the final stages of purification necessary to ensure the elimination of bacteria and bacterial endotoxins [1].

\section{Types of waters}

Purified Water (PW), Highly Purified Water (HPW), and Water for Injection (WFI) used in pharmaceutical processes are produced on site from the local potable water, which has been produced by the treatment of the feed water [2].

PW: tis a water that is obtained from drinking water, it is used as an excipient in the formulation of non-sterile pharmaceutical products and must comply with the specifications established in the regulatory agencies. It is produced through systems that include processes such as, reverse osmosis, ultrafiltration $[1,11]$. PW is used as an excipient in liquid pharmaceutical forms and in the preparation of many of the solid forms, for the washing of equipment and as raw material for obtaining higher quality waters, these must not necessarily be sterile, nor are they exempt of pyrogens, the requirement of this is less than that dedicated to injection [20].

WFI: It is a sterile water that is used as an excipient in the production of finished pharmaceutical injectable forms and in the production of active pharmaceutical ingredients that require it. It is a critical component of sterile preparations intended for parenteral administration. Obtaining it is accepted by distillation and double-step reverse osmosis, although some regulatory agencies only allow the one obtained from the first case [20]. It is used in the washing of equipment used for sterile preparations depending on the immediate use of said water. PW and WFI systems must be validated and calibrated to produce and distribute water of acceptable microbiological and chemical quality in a reliable and regular manner [11].

\section{Guidelines Overview}

Understanding the water needs of a certain quality requires knowledge of the different water quality standards and the corresponding organizations that establish it. Determining the correct standard to follow depends on the regulatory environment in which the facility operates and the specific applications at each point of use. The specifications for the PW vary from one pharmacopoeia to another, both in determinations to be made and in established limits, even for obtaining methods; this aspect that makes regulatory compliance a bit more complex for companies that have a broad market for their products. In the case of the WFI there is greater harmonization in terms of limits, but there are still differences in the determinations to be made as well as the method of obtaining said water quality. For PW recognized methods of obtaining are similar, but there is disparity in determinations to be made and the limits for these. In WFI the opposite occurs, there is a divorce between the approved generation methods, but they are more similar to the established limits. For example, USP allows WFI produced by distillation or another equivalent process, the Japanese pharmacopoeia does not accept that equivalent process and does recognize ultrafiltration and EP only allows distillation $[10,11,21]$.

Organizations such as the American Society for Sampling and Materials (ASTM), the College of American Pathologists (CAP), the International Organization for Standardization (ISO), the National Committee of Clinical Laboratory Standards (NCCLS) and the United States Pharmacopeia (USP), publish these standards. Commonly, industries tend to be guided by the organization to which they are affiliated. USP 40 states that the PW is obtained from water that complies with the National Primary Drinking Water Regulation of the US Environmental Protection Agency, regulations for drinking water of the European Union or Japan or with the Guidelines for the Quality of Drinking Water of the WHO [11]. The USP 40, and EP 9 have adopted similar standards for the quality (conductivity) of Bulk Pharmaceutical Waters (Table 2) [22]. 
Table 2: Standards of water [22].

\begin{tabular}{|c|c|c|c|c|}
\hline \multirow{2}{*}{ Parameter } & \multicolumn{2}{|c|}{ PW } & \multicolumn{2}{c|}{ WFI } \\
\cline { 2 - 5 } & USP & EP & USP & EP \\
\hline $\begin{array}{c}\text { Conductivity at } \\
20^{\circ} \mathrm{C}\end{array}$ & $\mathrm{N} / \mathrm{A}$ & $\leq 4.3 \mu \mathrm{S} / \mathrm{cm}$ & $\mathrm{N} / \mathrm{A}$ & $\begin{array}{c}\leq 1.1 \mu \mathrm{S} / \\
\mathrm{cm}\end{array}$ \\
\hline $\begin{array}{c}\text { Conductivity at } \\
25^{\circ} \mathrm{C}\end{array}$ & $\leq 1.3 \mu \mathrm{S} / \mathrm{cm}$ & $\mathrm{N} / \mathrm{A}$ & $\begin{array}{c}\leq 1.3 \mu \mathrm{S} / \\
\mathrm{cm}\end{array}$ & $\mathrm{N} / \mathrm{A}$ \\
\hline
\end{tabular}

N/A: Not an applicable requirement.

USP is governed by the pharmaceutical industry and ISO is governed by quality organizations from multiple industries [23]. The qualities of PW and WFI are described and recognized in the pharmacopoeias, while the HPW is a classification of the EP and the WHO, and only differs from the WFI by the obtaining method. The ICH (International Conference on Harmonization) exists with the objective of harmonizing international regulations, and some steps have been taken, but as far as pharmaceutical waters are concerned, little progress has been made. The USP has a discussion group that since 2004 initiated a project to try to harmonize the requirements of pharmaceutical waters between USP, EP and JP (Japanese Pharmacopeia) [24,25]. Table 2 shows the upper limits of conductivity allowed by the USP and the EP for different temperatures $[9,12,14,26]$.

\section{Conductivity Channel Calibration for Pharmaceutical Waters Systems}

The metrological characteristics of a measuring instrument can be very varied, depending on the measurement process which are decisive. In the calibration for physical/electrical magnitudes, such as conductivity, some of typical uncertainty sources are: resolution, hysteresis, precision repeatability, stability, accuracy, maximum permissible error (the linearity, trazability, hysteresis, drift, repeatability, accuracy, etc. are specified by the manufacturer within this definition in several cases).

Depending on the applications, users validate their procedures, calibrate the instruments and perform additional instrument controls, such as system aptitude tests and quality control check samples, guaranteeing that the acquired data are reliable. The calibration is the operation that under specified conditions establishes, in a first stage, a relationship between the values and their associated measurement uncertainties obtained from the measurement standards, and the corresponding indications with their associated uncertainties and, in a second stage, uses this information to establish a relationship that allows a measurement result from an indication [27]. The conductivity calibration procedures in pharmaceutical water systems is constantly re-evaluated [28].

\section{Instrumentation and reference materials certified (CRM)}

Every calibration process requires reference materials, standards and instrumentation for its development. A Reference
Material is a sufficiently homogeneous and stable whit his specified properties [16,17]. A Certified Reference Material (CRM) is a reference material accompanied by documentation issued by an authorized institution, which provides one or more values whit specified properties, uncertainties and traceabilities associated, using validated procedures. Traceability is an important metrological characteristic, it is the property of the result of a measurement to be related to a metrological reference established through an uninterrupted chain of calibrations or comparisons [6].

\section{CRM for electrode calibration}

a. Standard solution (SS)

b. Thermometer: which is inserted in SS (temperature calibration only) [16].

\section{CRM for conductimeter calibration (full channel)}

I. Resistor sets, a decadic box, or even a variable resistance device can also be used for comparison between the resistance value indicated by the conductimeter and the certified value of each standard resistor.

\section{Regulatory issues}

In chapter $<645>$ of USP 40 the criteria of some global pharmacopoeias are harmonized. The requirements for conductivity calibration include the conductimeter and electrode. Due to the characteristics of the SS that is used for conductivity calibration in pharmaceutical waters systems, the conductivity cell must be 4-pole. Only this type of cell can guarantee a perfect linearity, thanks to the non-polarization of the electrodes (poles)

\section{USP requirements for the conductimeter [16-17]}

I. Report uncompensated conductivity.

II. Display resolution of $0.1 \mu \mathrm{S} / \mathrm{cm}$ mínimum.

III. Temperature measurement circuit should be verified.

IV. Calibration (or verification) of the resistance measurement is accomplished by replacing the conductivity sensor electrodes with precision resistors having standards traceable to NIST or equivalent national authorities in other countries (accurate to $\pm 0.1 \%$ of the stated value) to give a predicted instrument conductivity response. The accuracy of the resistance measurement is acceptable if the measured conductivity with the traceable resistor is within $\pm 0.1 \mathrm{mS} /$ $\mathrm{cm}$.

\section{USP requirements for the sensor $[16,17]$}

i. Cell constant is accurate and known to $\pm 2 \%$.

ii. Calibrate sensor in a solution with a stated.

iii. Calibrate sensor in a solution prepared to a specific conductivity (ASTM D1125 standard or ultrapure water) 


\section{Global Journal of Pharmacy \& Pharmaceutical Sciences}

iv. Calibrate sensor vs. another calibrate sensor (typically from manufacturer)

v. Temperature accurate to $2^{\circ} \mathrm{C}$, effective USP $40[7,11,16]$.

EP requirements for meter and sensor $[7,16]$

a) Temperature

Accuracy $\pm 2{ }^{\circ} \mathrm{C}$

b) Conductivity sensor cell constant

Accuracy $\pm 2 \%$, or re-calibrate if outside this range

$<1500 \mu \mathrm{S} / \mathrm{cm}$ calibration solution or by comparison with a sensor of a certified cell constant "Verified at suitable intervals"

c) Conductivity meter

Accuracy $\pm 0.1 \mu \mathrm{S} / \mathrm{cm}$ or better

Use certified precision $0.1 \%$ resistors

d) System calibration

Only if in-line sensors cannot be dismantled

Against one or more certified solutions

Accuracy of $\pm 3 \%$ of measured conductivity $+0.1 \mu \mathrm{S} / \mathrm{cm}$.

\section{Uncertainty}

Uncertainty as a scientific term does not refer to the terms error or mistake, but rather to the variability implicit in the measurements, and that cannot be eliminated, only estimated and made sure that it is as small as possible. The procedure success depends critically on the specialist who performs the analysis of the sources of the uncertainty and the ability to convince others of this understanding [29]. It is important to distinguish the difference between error and uncertainty. The error is defined as the difference between an individual result and the conventional value of the measurand. As such, the error is a single value. At first, the value of the known error can be applied as a correction to the result. On the other hand, the uncertainty takes form of an interval and is estimated for an analytical procedure. Generally, the value of the uncertainty cannot be used to correct the result of the measurement [30]. However, the error analysis modifies and contributes to the measurement uncertainty.

The uncertainty is a non-negative parameter, which characterizes the dispersion of the values attributed to a measurand, based on the information used [31]. The standard uncertainty is the uncertainty of the measurement result expressed as a standard deviation [32]. It can also be classified as a parameter associated with the result of the measurement that characterizes the dispersion of values that can be reasonably attributed to the measurand [30]. The uncertainty of the measurement includes components from systematic effects associated with corrections and values assigned to patterns, as well as the uncertainty due to the definition. Sometimes the estimated systematic effects are not corrected and instead are treated as components of uncertainty. Usually, for a given information, it is understood that the uncertainty of the measurement is associated with a certain value attributed to the measurand. Therefore, a modification of this value supposes a modification of the associated uncertainty [31].

The uncertainty is quantified by the standard deviation of the probability distribution of the measurand. A conventional way of classifying uncertainties is recommended according to how the estimation of the probability distribution of the measurand is carried out. Hence, there will be: Type A and Type $B$ evaluation. The purpose of the Type A and Type B classification is to indicate the two different ways to evaluate components of uncertainty; the classification does not mean that there is any difference in the nature of the components that result from each of the two types of evaluation. Both types of evaluation are based on probability distributions, and the components of the resulting uncertainty of any type are quantified by variances and standard deviations. The selection of the Type A uncertainty can give information on the characteristics of the measurand, but the quality of it depends on the number of observations $\mathrm{n}$, while a Type B evaluation of an uncertainty component is generally based on a common source of information comparatively trustworthy. This information may include; data from previous measurements, experience with general knowledge of the characteristics, behavior and properties of relevant materials and instruments, specifications of manufacturers, data obtained from both calibration certificates and other types of certificates, uncertainties assigned to data from reference taken from manuals among others [31,33]. Figure 5 contains the proposed distributions to evaluate the calibration uncertainty in the conductivity channel for pharmaceutical waters systems [2834].

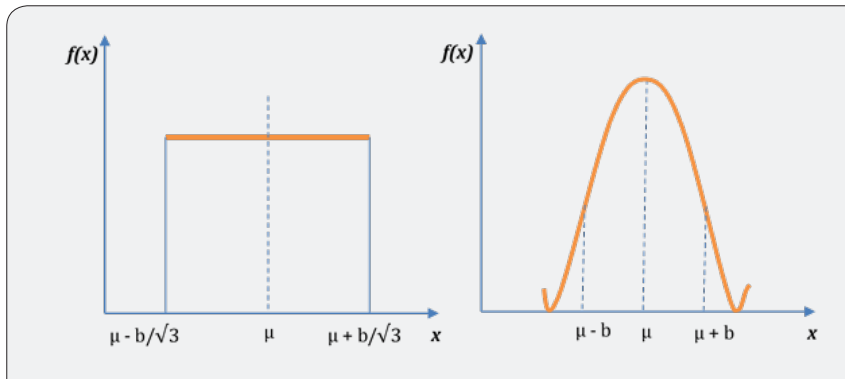

Figure 5: Typical uncertainty assessment by Type B approximation. a) Rectangular distribution; for accuracy, resolution, drift, etc. Although the resolution given by the value of the last digit of the indicator is $\delta \mathrm{x}$ and is expressed as the variance of the distribution $\mathrm{u}^{2}=(\delta \mathrm{x}) 2 / 12$. b) Normal distribution; for repeatability, reproducibility, etc. [28,34].

Table 3 shows the different mathematical approximations for each uncertainty. The rectangular distribution is the right way for uncertainty quantification in the conductivity channel calibration, thus foreseeing the worst case (maximum uncertainty interval) [33]. The typical uncertainty, when this result is obtained from the values of a set of other quantities, is called Combined Typical Uncertainty $\left(\mathrm{u}_{\mathrm{c}}\right)$, that is; the uncertainty of the measurand $\mathrm{Y}$ leads to an assessment of its combined 
Typical Uncertainty $\left(u_{c}(y)\right)$, obtained from other magnitudes [31]. The $u_{c}(y)$ of the result of a measurement is equal to the positive square root of a sum of terms, these terms being the variances and covariances of these other magnitudes weighted according to how the measurement result varies with respect to changes in these magnitudes, the so-called law (Taylor's series) of propagation of uncertainties. The $u_{c}(y)$ must be expressed by the numerical value obtained by applying the usual method of combination of variances. The $\mathrm{u}_{\mathrm{c}}(\mathrm{y})$ and its components should be expressed in the form of "standard deviations" [33,35].

Table 3: Probability distributions according to the Type B evaluation [33].

\begin{tabular}{|c|c|c|}
\hline About the Magnitude & Probability Distribution & $\begin{array}{l}\text { Typical Uncertainty } \\
\text { u(xi) }\end{array}$ \\
\hline $\begin{array}{l}\text { CRM certificate. Express an uncertainty } U \text { equal to k times the } \\
\text { standard deviation. }\end{array}$ & The assumed by the signer of the certificate & $\frac{U}{k}$ \\
\hline $\begin{array}{l}\text { Calibration certificate expresses a confidence interval } \Delta x i \\
\text { corresponding to a level } \mathrm{p} \text { and freedom degree } v \text {. }\end{array}$ & Normal distribution & $\begin{array}{c}\frac{\Delta X i}{t p} \\
\mathrm{t}_{\mathrm{p}}(v)-\text { Student coefficient }\end{array}$ \\
\hline $\begin{array}{l}50 \% \text { of probability that } \mathrm{Xi} \text { is in interval of }-\mathrm{b} \text { to }+\mathrm{b} \text {. The values are } \\
\text { most probables in the proximity to the average value. }\end{array}$ & Normal distribution & $\begin{array}{c}1.48 \times b \\
b=\frac{(+b)-(-b)}{2}\end{array}$ \\
\hline $\begin{array}{l}2 / 3 \text { of probability that } \mathrm{Xi} \text { value is in interval of }-\mathrm{b} \text { to }+\mathrm{b} \text {. The values } \\
\text { are most probables in the proximity to the average value. }\end{array}$ & Normal distribution & $\mathrm{b}$ \\
\hline $\begin{array}{l}\text { The probability that Xi value is in interval of }-\mathrm{b} \text { to }+\mathrm{b} \text { is equal to } 1 \text { and } \\
\text { that it is outside is } 0 \text {. Any point of the interval is equiprobable. }\end{array}$ & Rectangular distribution & $\frac{b}{\sqrt{3}}$ \\
\hline $\begin{array}{l}\text { The probability that Xi value is in interval of }-b \text { to }+b \text { is equal to } 1 \text { and } \\
\text { that it is outside is } 0 \text {. Central values are most probables. }\end{array}$ & Triangular distribution & $\frac{b}{\sqrt{6}}$ \\
\hline $\begin{array}{l}99.73 \% \text { of probability that Xi value is in interval of }-\mathrm{b} \text { to }+\mathrm{b} \text {. Central } \\
\text { values are most probables. }\end{array}$ & Normal distribution & $\frac{b}{3}$ \\
\hline
\end{tabular}

$$
u_{c}^{2}(y)=\sum_{i=1}^{N} u_{i}^{2}(y)+2 \sum_{i=1}^{N-1} \sum_{k=i+1}^{N} u_{i}(y) u_{k}(y) r\left(x_{i}, x_{k}\right)
$$

Where:

$u_{i}^{2}(y)=c_{i}^{2} u^{2}\left(x_{i}\right), u_{i}^{2}$ is the contributions of the variances of each input quantity to the measurement system. Besides, $c_{i} \frac{\delta f}{\delta x}, c_{i}$ is the sensitivity coefficient, which is numerically calculated by substituting $u_{i}^{2} \mathrm{u}^{2}$ by Zi.

$$
Z_{i}=\frac{1}{2}\left[f\left(x_{i}, \ldots, x_{i}+u\left(x_{i}\right), \ldots x_{N}\right)-f\left(x_{i}, \ldots, x_{i}+u\left(x_{i}\right), \ldots x_{n}\right]\right.
$$

The partial derivatives $\left(\frac{\delta f}{\delta x}\right)$ of input are equal to the output derivatives for $\mathrm{Xi}=\mathrm{xi}$ (in practice, the partial derivatives are

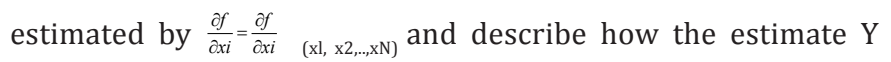
varies with the changes in the estimates of the arguments $\mathrm{xl}$, $\mathrm{x} 2, . ., \mathrm{xN}$. This coefficient can also be obtained experimentally. On the other hand: $r$ is the correlation coefficient that expresses the relationship that exists between the inputs and outputs of the calibration system.

$$
r\left(x_{i}, x_{k}\right)=\frac{u\left(x_{i}, x_{k}\right.}{u\left(x_{i}\right) u\left(x_{k}\right)}
$$

If non-correlation is demonstrated, from equation 1 ; will be defined:

$$
u_{c}(y)=\sqrt{\sum_{i=1}^{N} c_{i}^{2}} u^{2}\left(x_{i}\right)
$$

Adapted (from the equation 2) to the conductivity calibration procedure for pharmaceutical waters systems:

utotal combined $=\left(\mathrm{u}^{2} \mathrm{CRM}+\mathrm{u}^{2}\right.$ conductimeter + electrode + $\mathrm{u}^{2}$ calibration procedures $+\mathrm{u}^{2}$ environmental conditions $+\mathrm{u}^{2}$ data processing $+u^{2}$ operator $+u^{2}$ temperature contribution* ${ }^{*} 1 / 2$

* This contribution is the biggest difference between conductivity values (attached table in the CRM) for a temperature change equal to the resolution of the instrument [36]:

$\frac{\text { Temp. minimum charge (attached in CRM) }}{\text { conuctimeter Temp. resolution }}=\frac{\text { conduct. minimum change (attached in CRM) }}{\mathrm{U}_{\text {emperasur corribution }}}$

To satisfy the needs of some industrial and commercial applications, as well as the requirements in areas of health and safety, an Expanded Uncertainty $\mathrm{U}=\mathrm{k} \times \mathrm{u}_{\mathrm{c}}(\mathrm{y})$ is obtained by multiplying the $\mathrm{u}_{\mathrm{c}}(\mathrm{y})$ by a coverage factor $\mathrm{k}$, which is a number greater than one [33]. The purpose of obtaining $U$ is to provide an interval around the result of a measurement in which we can expect to include a significant fraction of the distribution of values that can reasonably be attributed to the measurand. The choice about $\mathrm{k}$, is based on the level of confidence required for the interval, which usually is in the range of 2 to 3 which in turn gives the approximate $95 \%$ to $99 \%$ probability that the measurand is in the environment $(\mathrm{y}-\mathrm{U} ; \mathrm{y}+\mathrm{U})$. The coverage factor must always be declared, in such a way that the typical uncertainty of the measurand can be recovered for use in the calculation of the $u_{c}(y)$ of other measurement results that may depend on that quantity $[33,35]$.

\section{Conformity assessment}

Conformity assessment in a wide sense is defined as any activity performed in order to obtain by direct or indirect ways 


\section{Global Journal of Pharmacy \& Pharmaceutical Sciences}

if any product, process or system accomplish some defined requirements or rules [33]. Measurements are main sources of information to define whether any product is compliance or not. In the evaluation of conformity, the acceptance limits of the product and/or service must be determined to ensure that a desired probability of conformity is obtained through a measurement made of the characteristic of the finished object. This limit may respond to quality or regulatory requirements, the conformity evaluation is basically any activity related to direct and indirect determination of accomplishment in requirements. In regulatory context are defined requirements of security, efficacy and effectivity.

The instrument calibration is a kind of measurement, expressing the real values as a probability density function (PDF) or as a numerical approximation function. Due to the fact that measurement never gives a complete information there is always risk of mistakes when deciding if the measurement is conforming or not conform related to some requirement. This mistake has two nuances: accepting a not conform measurement or rejecting a conform measurement. If the real value in any measurement is contained within the permitted values (TI) then the measurement process is conforming, any other way is not conform [2].

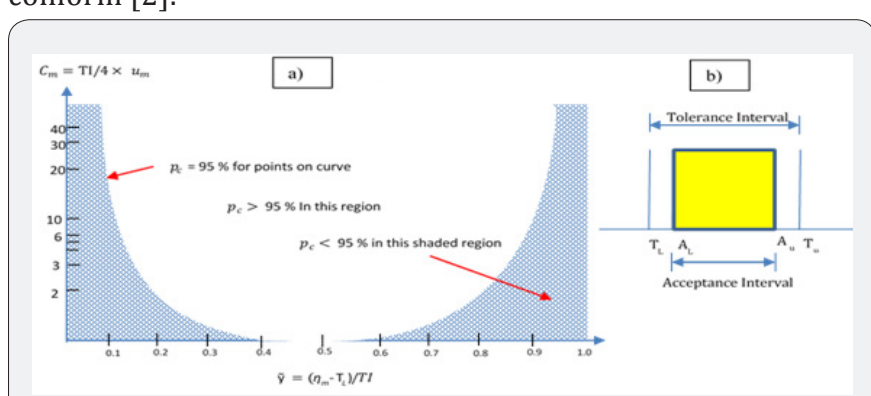

Figure 6 : a) Showing the locus of constant $95 \%$ conformance probability. The curve separates regions of conformity and nonconformity at a $95 \%$ level of confidence b) Binary conformity assessment where decisions are based on measured quantity values. The true value of a measurable property (the measurand) of an item is specified to lie in TI denfied by limits (TL; TU). The item is accepted as conforming if the measured value of the property lies in an Al defined by limits (AL; AU) and rejected as non-conforming otherwise [33].

The acceptance limit (AI) is the higher or lower of measured values permitted in the characteristic and the TI is higher $\mathrm{T}_{U}$ or lower $\mathrm{T}_{\mathrm{L}}$ value of real values in a characteristic [33]. Tolerances cannot be seen as uncertainties. The AI chosen to any process are not uncertainties either, all specifications define what is desired of a product. The chosen values of TI and $\mathrm{AI}$ is related to consequences of deviation in quality of desired measure process [2]. An AI contains the set of true values of a measurand with a given probability, based on the available information (Figure $6)$. The AI can be obtained from an expanded uncertainty $\left(u_{m}\right)$ [33]. Another related definition in conformity assessment is the Measurement capacity index $(\mathrm{Cm})$, defined in Figure 6 as well $[35,33]$.

The interval $\left(y-2 u_{m}\right.$; and $\left.+2 u_{m}\right)$ must contain a significant fraction of the distribution of values that can reasonably be attributed to Y. The PDF of a measurement characterizes the $\eta \mathrm{m}$ possible values that the measurement can take. The $p_{\varepsilon}$ can be expressed as a function of TL and the particular result of the measurement expressed as ( $y, u)$, which in this case would be $\mathrm{y} \approx \eta \mathrm{m}$ and $\mathrm{u} \approx \mathrm{um}$. From here; a new term $\mathrm{y}$ is defined, which characterizes the proximity of the measurement to its tolerance limits [33]. The smaller $\mathrm{C}_{\mathrm{m}}$ is the narrower the interval to obtain a $p_{c}$ greater than or equal to $95 \%$. In the biopharmaceutical industry, the $p_{c}$ must be greater than or equal to $95 \%$. [37].

\section{Concluding Remarks}

Although the highest conductivity value for PW and WFI does not exceed $10 \mu \mathrm{S} / \mathrm{cm}$ (Table 4), currently, a CRM of this order can't be produced with an acceptable uncertainty. The SS conductivity value must be higher than that the operation point, as the EP proposes $(<1500 \mu \mathrm{S} / \mathrm{cm})$, because an SS of low conductivity values and with a U lower than $1 \%$ is impossible to achieve, according to recent studies by NIS [38]. Neither should a CRM be used that has remote conductivity values with respect to the set point of the process, so a commitment relationship it's established. For the conductivity channel calibration in the pharmaceutical waters systems the greatest source of uncertainty is the accuracy of the electrode cell constant, while for temperature; the greatest uncertainty contribution is provided by the conductimeter accuracy [36]. In this paper, the criteria of pharmacopoeias and regulatory agencies about calibration, the role of temperature and the conformity assessment in the conductivity measurement channels in pharmaceutical waters systems are harmonized.

Table 4: Temperature and conductivity requirements (for non-temperature-compensated conductivity measurements only) $[9,12,14,26]$.

\begin{tabular}{|c|c|c|c|c|c|}
\hline \multirow{2}{*}{ Temperature $\left({ }^{\circ} \mathrm{C}\right)$} & \multicolumn{2}{|c|}{ Limit of Conductivity $(\mu \mathrm{S} / \mathrm{cm})$} & \multirow{2}{*}{$\begin{array}{c}\text { Temperature } \\
\left({ }^{\circ} \mathrm{C}\right)\end{array}$} & \multicolumn{2}{|c|}{ Limit of Conductivity $(\mu \mathrm{S} / \mathrm{cm})$} \\
\hline & WFI & PW & & WFI & PW \\
\hline 0 & 0.6 & 2.4 & 50 & 1.9 & 7.1 \\
\hline 5 & 0.8 & - & 55 & 2.1 & - \\
\hline 10 & 0.9 & 3.6 & 60 & 2.2 & 8.1 \\
\hline 15 & 1 & - & 65 & 2.4 & - \\
\hline 20 & 1.1 & 4.3 & 70 & 2.5 & 9.1 \\
\hline 25 & 1.3 & 5.1 & 75 & 2.7 & 9.7 \\
\hline 30 & 1.4 & 5.4 & 80 & 2.7 & 9.7 \\
\hline
\end{tabular}




\section{Global Journal of Pharmacy \& Pharmaceutical Sciences}

\begin{tabular}{|c|c|c|c|c|c|}
\hline 35 & 1.5 & - & 90 & 2.7 & 9.7 \\
\hline 40 & 1.7 & 6.5 & 95 & 2.9 & - \\
\hline 45 & 1.8 & - & 100 & 3.1 & 10.2 \\
\hline
\end{tabular}

\section{References}

1. Bermúdez YH, Rodríguez DE (2006) Calificación de los sistemas de generación, almacenamientoy distribución de agua purificada y agua para inyección del Centro de Inmunológica Molecular. Facultad de Ingeniería Química, CUJAE, Habana, Cuba.

2. Ginett Vargas Hoyos GGH (2013) Evaluación de la Conformidad de la Calibración de un Cromatógrafo Líquido de Alta Resolución.

3. (2017) Measuring the Conductivity of Pure Water Using USP $<645>$. Thermo Scientific, USA.

4. Mettler-Toledo Thornton I (2009) 200CR Two-Channel Conductivity/ Resistivity Measurement System.

5. FDA (1986) Water for Pharmaceutical Use.

6. BIPM I, IFCC, ILAC, ISO, IUPAC, et al. (2012) Evaluation of measuremen data-The role of measurement uncertainty in conformity assessment.

7. Modla M (2011) USP 645: in line measurement of water conductivity.

8. Dalmas P (2000) Conductivity measurement on pure water according to the recommendations of the USP Pharmacopoeia USP24-NF19.

9. Conductivity RASA (2005) Theory and Practice.

10. (2017) European Pharmacopeia $9^{\text {th }}$.

11. USP 40 NF 35 (2017) Farmacopea 35.

12. Reinhard MJS (2007) Information on Conductivity Measurement.

13. Braga VM (2004) Conductivity Sensor Calibrations to Meet Water Industry Requirements.

14. (2013) Pharmaceutical Waters Guide for Regulatory Compliance, Analysis and Real-Time Release.

15. Bevilacqua A (2010) Instrumentation for product control vs. process control-Which features are relevant for monitoring high-purity pharmaceutical water systems?

16. Management EP (2010) Theory and application of conductivity.

17. Bevilacqua AC (1999) Harmonization of Conductivity Tests for Pharmaceutical Waters.

18. Truman SL, Anthony C, Bevilacqua, Kenneth RM (2005) The Fundamental Conductivity and Resistivity of Water.

19. Bevilacqua AC (2002) Calibration and Performance of a Conductivity System to Meet USP 23*.

20. Dabbah R (2006) USP Pharmaceutical Waters, Part 1.

21.JPM Association (2017) Pharmaceutical Administration and Regulations in Japan.
22. Shukshith NVGKS (2016) Water for Pharmaceutical Use.

23. ICH/EMEA (1999) Test procedures and acceptance criteria for new drug substances and new drug products: chemical substances Q6A. Q6A Step 4, Reino Unido, UK, England, pp. 3-30.

24. Dabbah R (2006) USP Pharmaceutical Waters, Part 1. Bio Process International 4: 58-62.

25. Dubbah R (2006) USP Pharmaceutical Waters, Part 2. Bio Process International 4: 58-62.

26. Management EP (2005) Water for injection measurements.

27. Corporation L (2003) A guide to laboratory water purification.

28. FDA-ISPE (2001) Baseline: Pharmaceutical Engineering Guide.

29. Taylor JR (1997) An Introduction to error analysis. The study of uncertainties in phisical measurements. ( $2^{\text {nd }}$ edn), University of Colorado, Colorado, USA, p. 327.

30. Ellison ELR (2000) EURACHEM/CITAC, EURACHEM/CITAC Guide Quantifying Uncertainty in Analytical Measurement. (2 ${ }^{\text {nd }}$ edn), QUAM:2000.1, LGC, UK, England, pp. 1-126.

31. JCGM (2008) International vocabulary of metrology-Basic and general concepts and associated terms (VIM). JCGM 200:2012, Pavillon de Breteuil, Paris, Italy, p. 108.

32. WHO (2016) WHO Expert Committee on Specifications for Pharmaceutical Preparations. Technical Report Series, OMS, Ginebra, Suiza.

33. JCGM (2012) Evaluation of measurement data-The role of measurement uncertainty in conformity assessment. JCGM 106:2012, Pavillon de Breteuil, Paris, Italy, p. 5-7.

34.FDA (2008) Guidance for Industry Process Validation: General Principles and Practices.

35. JCGM (2008) Evaluation of measurement data-Guide to the expression of uncertainty in measurement. Pavillon de Breteuil, Paris, Itlay, p. $5-134$.

36. Adolfo Castillo Caballero GVH (2017) Evaluación de conformidad en la calibración del canal de medición de conductividad para aguas farmacéuticas. Vacci Monitor 3: 93-101.

37. ISO/IEC (2017) Evaluación de la conformidad. Requisitos generales para la competencia de los laboratorios de ensayo y de calibración.

38. Ramos DA (2012) Desempeño de los servicios de aguas farmacéuticas para la producción de anticuerpos monoclonales en la planta Antyter del CIM. Maestria, CIM, Habana, Cuba.
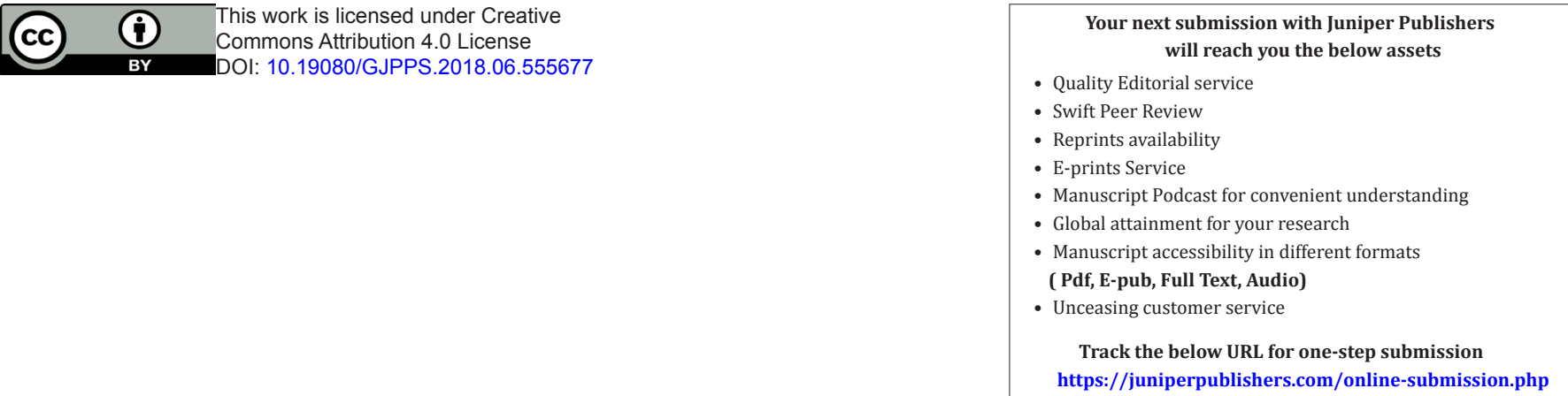